\title{
A case of DENV and SARS-CoV-2 coinfection in Indonesia The challenge of a dual outbreak?
}

\author{
A Christanto $^{1}$, AS Listyoko $^{1}$, IN Chozin $^{1}$ \\ Sri Lankan Journal of Infectious Diseases 2020 Vol.10(2):155-161
}

DOI: http://dx.doi.org/10.4038/sljid.v10i2.8306

\begin{abstract}
Indonesia, as the world does, is currently facing the coronavirus disease 2019 (COVID-19) pandemic. Indonesia is a tropical, dengue virus (DENV)-endemic country where year-long cases of DENV occur, particularly in the rainy season. Thus, the country is on the brink of facing the "dual outbreak" of COVID-19 and dengue fever (DF). We report a case of coinfection between SARS-CoV-2 and DENV in a 39-year-old man initially diagnosed with DENV infection and with no suspicion of COVID-19, who later developed respiratory symptoms. Further tests confirmed the SARS-CoV-2 infection, and the patient was finally diagnosed with COVID-19 and DF coinfection. As both viruses may possess similar clinical and laboratory profiles at the time of presentation, it is important to recognize the clues of single and coinfection by the two viruses to ensure timely management, particularly in patients with COVID-19.
\end{abstract}

Keywords: COVID-19, SARS-CoV-2, DENV infection, coinfection

\section{Introduction}

At the end of December 2019, China reported a series of patients with pneumonia of unknown origin. Within 3 days, the case number rose to 44. Isolates from patients showed signs of an infection with a novel coronavirus, which at that time was named as 'novel coronavirus 2019' (2019-nCoV). On February $11^{\text {th }}$ 2020, the World Health Organization (WHO) named the new virus as SARS-CoV-2 for its resemblance to SARS-CoV which caused the 2002 SARS outbreak and the disease itself as coronavirus disease 2019 (COVID-19). ${ }^{1}$ On March $11^{\text {th }} 2020$, WHO declared COVID-19 as a pandemic. ${ }^{2}$ In Indonesia, the pandemic started on March $2^{\text {nd }}$ 2020, with the index case identified in the country's capital, Jakarta. The pandemic reached the whole country by the end of April 2020. Currently, more than 25,000 positive cases have been identified in Indonesia, with a mortality rate of about $6 \%$. However, the trend is still increasing. ${ }^{3}$

\footnotetext{
${ }^{1}$ Department of Pulmonology and Respiratory Medicine, Brawijaya University Address for correspondence: Christanto A, Saiful Anwar General Hospital, Jl. Jaksa Agung Suprapto No.2, Malang, Indonesia. Telephone: +6281358591991E-mail: anthonychris@student.ub.ac.id

https://orcid.org/0000-0002-5175-8212

Received 22 June 2020 and revised version accepted 21 September 2020 
Dengue virus (DENV) is a virus of the genus Flaviviridae responsible for dengue fever. It is the most important arthropod-borne virus infection in the world, with a mortality rate of more than 25,000 deaths anually. ${ }^{4}$ Indonesia is a tropical country where both vector mosquitos of DENV, Aedes aegypti and Aedes Albopictus are endemic in almost the whole country. The country is now facing a rising number of DENV infection. ${ }^{5}$ The country's ministry of health has reported more than 50,000 cases of DENV infection across the country as of May $16^{\text {th }} 2020$ with a total number of deaths reaching more than 300 . The country is therefore facing the socalled "dual outbreak" as the rainy season of the year is approaching in the following months.

It is difficult to distinguish DENV and SARS-CoV-2 infection, as both share similar clinical and laboratory features, as well as the disease course, particularly in DENV-endemic areas. The appearance of atypical symptoms of COVID-19, which may be confused as a DENV infection, may therefore delay the correct diagnosis and treatment. ${ }^{6}$ Here, we report a case of DENV and SARS-CoV-2 coinfection in our tertiary hospital in Malang regency, East Java, Indonesia. It is important to note that East Java is the province with the highest number of COVID-19 cases in Indonesia as of May $29^{\text {th }} 2020$ and also reports about 3,600 cases of DENV infection as of April $22^{\text {nd }} 2020$.

\section{Case History}

A 39-year-old man was referred to our hospital with suspected COVID-19. He was admitted five days prior to the referring hospital with chief complaints of intermittent fever for one week prior to referral, accompanied by headache and myalgia. He developed a dry cough 4 days prior to the referral which was worsening over time. The patient was a public civil servant and had no febrile contact in his household. He had neither a history of recent travel outside of the city nor contact with confirmed or a possible case of COVID-19. A chest CT scan was then arranged due to his worsening cough.

The chest CT showed bilateral ground glass opacities mainly in the peripheral lung area with reactive lymphadenopathy (figure 1), which was labeled as 'probable COVID-19' by the radiologist. The patient was then referred to our hospital for further testing and treatment.

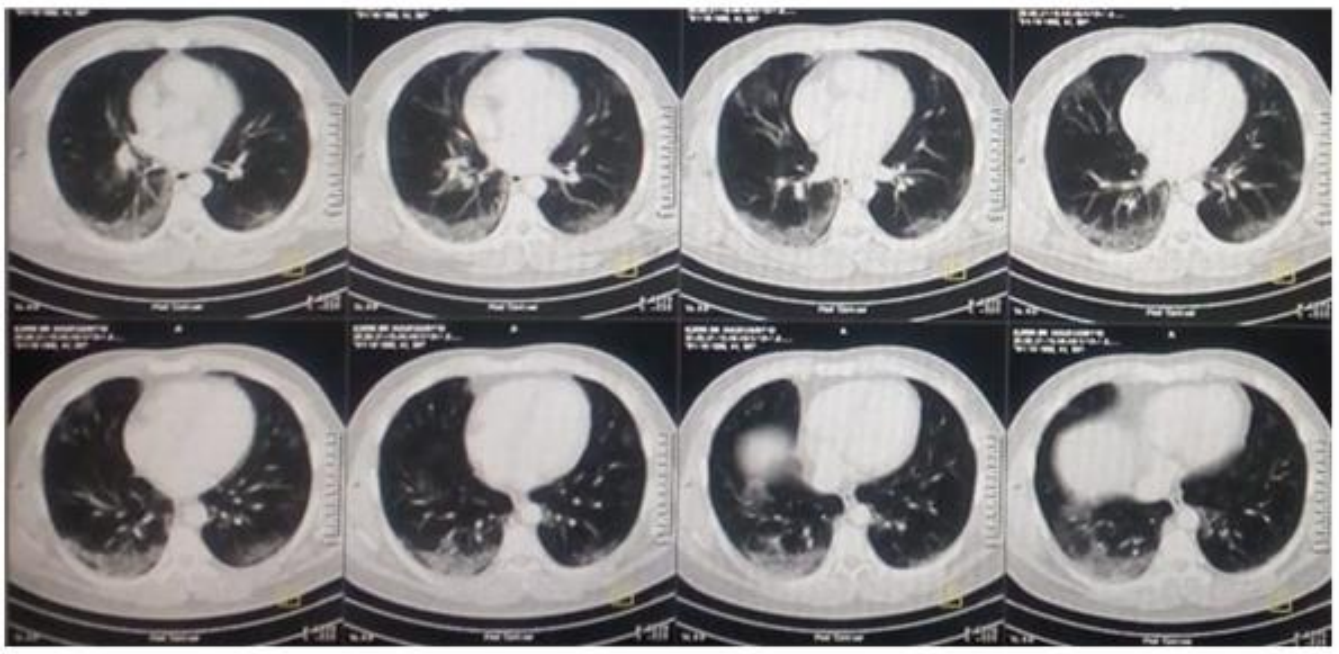

Fig 1. CT Scan of the patient in May $21^{\mathrm{st}}, 2020$, showing bilateral ground glass opacities predominantly in peripheral lung area. 
Initial findings in our hospital included symptoms of fever, dry cough, nonspecific headache, and myalgia, but with no breathing difficulties or loss of smell/taste. There were no gastrointestinal symptoms or neurological impairment. Physical examination was within normal limits, with peripheral oxygen saturation of $97 \%$ in room air and respiratory rate of 18 /minute. He had no skin rashes or bleeding manifestations. Abnormal laboratory findings included elevated hemoglobin and hematocrit, elevated CRP, slightly elevated LDH, and elevated lactic acid, fibrinogen, and ferritin levels. Platelets were within the lower normal limit, despite the history of thrombocytopenia in the previous hospital (platelet count 112.000/uL) 4 days prior to the referral. The laboratory profile of the patient after admission is shown in Table 1.

Table 1. Laboratory profiles of the patient

\begin{tabular}{|c|c|c|c|c|c|}
\hline Laboratory Test & Onset D+7 & Onset D+9 & Onset D+12 & Onset D+15 & Normal Values \\
\hline Hemoglobin (g/dL) & 16.2 & 15.7 & 16.6 & 15.60 & $11.4-15.1$ \\
\hline Hematocrit (\%) & 45 & 44.1 & 48.4 & 43.8 & $38-42$ \\
\hline WBC $(/ \mu \mathrm{L})$ & 6,240 & 8,980 & 7,390 & 7,010 & $4,700-10,300$ \\
\hline Lymphocyte $(/ \mu \mathrm{L})$ & 1,379 & 2,289 & 1,840 & 2,347 & $>1200$ \\
\hline Platelets $(/ \mu \mathrm{L})$ & 187,000 & 254,000 & 369,000 & 438,000 & $142,000-424,000$ \\
\hline CRP (mg/dL) & 5.65 & 5.63 & 2.44 & 0.31 & $<0.3$ \\
\hline LDH (U/L) & 782 & 708 & 789 & 487 & $240-480$ \\
\hline Lactic acid (mmol/L) & 2.4 & & & & $0.5-1.6$ \\
\hline Fibrinogen (mg/dL) & & 433.5 & & & 154.3-397.9 \\
\hline Ferritin (ng/mL) & 1,388 & 1,284 & 1,302 & 1,250 & $30-400$ \\
\hline D-dimer (mg/L) & & 0.56 & 1.24 & 0.52 & $<0.5$ \\
\hline $\begin{array}{l}\text { Serum Ureum } \\
(\mathrm{mg} / \mathrm{dL})\end{array}$ & 12.4 & 10.0 & 10.6 & 14.5 & $16.6-48.5$ \\
\hline $\begin{array}{l}\text { Serum Creatinine } \\
(\mathrm{mg} / \mathrm{dL})\end{array}$ & 0.76 & 0.83 & 0.97 & 0.96 & $<1.2$ \\
\hline ALT (U/L) & 40 & 33 & 30 & 25 & $0-40$ \\
\hline $\operatorname{AST}(\mathbf{U} / \mathbf{L})$ & 50 & 50 & 51 & 50 & $0-41$ \\
\hline Serum Albumin (g/dL) & 4 & 4.27 & 4.48 & 4.23 & $3.5-5.5$ \\
\hline $\begin{array}{l}\text { Total Bilirubin } \\
(\mathrm{mg} / \mathrm{dL})\end{array}$ & 0.63 & 0.70 & 0.70 & 0.66 & $<1$ \\
\hline INR & & 1.04 & & 1.22 & $<1.5$ \\
\hline
\end{tabular}

The tests for DENV infection (DENV antibody test) in the referring hospital two days prior to the referral (5 days after onset of symptoms) showed positive results in both anti dengue $\operatorname{IgG}$ and IgM. A SARS-CoV-2 rapid antibody test was done in the referring hospital on the day of the referral (day 7 after onset of symptoms), which was negative.

SARS-CoV-2 testing was done using the GeneXpert巴 SARS-CoV-2 Xpress Assay on the day of the referral, with a positive result. The patient was diagnosed as having COVID-19 pneumonia and DENV infection. As per local protocol, we administered oxygen therapy with nasal cannula, maintenance fluid therapy using normal saline, antibiotic for prevention of secondary infection (moxifloxacin $400 \mathrm{mg} /$ day), vitamin C (400 mg/day), azithromycin (500 
mg tab/day), Hydroxychloroquine (400 mg/day), Oseltamivir (75 mg BID), and appropriate symptomatic therapy.

We rechecked the dengue antibody test on days 2 and 4 after admission ( 8 and 10 days after onset), with both tests showing positive results for both anti dengue IgM and IgG using the Panbio® Dengue Duo Casette. Routine laboratory tests and chest imaging using X-rays were performed every 3 days. Follow-up lab tests showed mild lymphocytopenia on day 9 and 12 after onset, increased D-dimer levels, and persistently high levels of ferritin. Second SARSCoV-2 GeneXpert ${ }^{\circledR}$ Xpress Assay test in 12 days after onset also showed positive result.

The patient underwent the first chest X-ray (CXR) 2 days prior to the referral; The initial CXR showed a wedge-shaped opacity in the right paracardial area suggestive of pneumonia. CXR on the day of referral was similar but with signs of infiltrates in the peripheral area of the left lung. CXR 3 days later showed improvement of the infiltrates (Figure 2a-c).

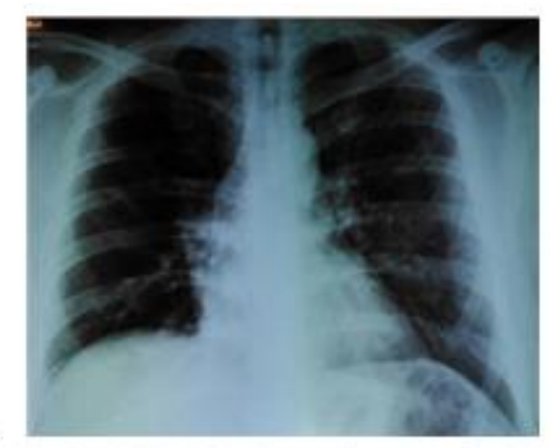

Fig 2. a) CXR 2 days prior to referral

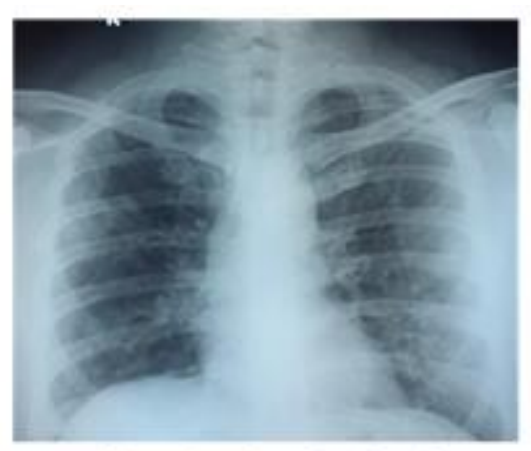

b) on the day of referral

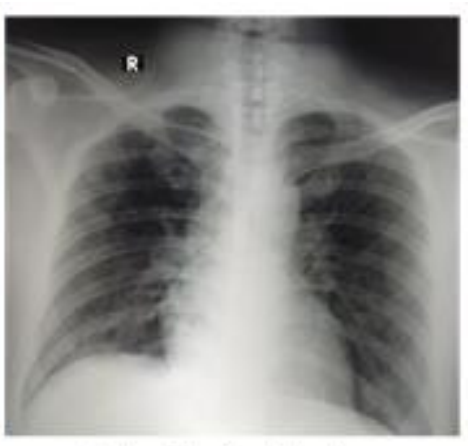

c) day 3 of admission

The last SARS-CoV-2 GeneXpert ${ }^{\circledR}$ Xpress Assay Result on day 14 after onset of symptoms remained positive. However, because the patient's symptoms had improved, the patient was discharged and referred to a private self-isolation facility on day 9.

The timeline is shown in Figure 3. 


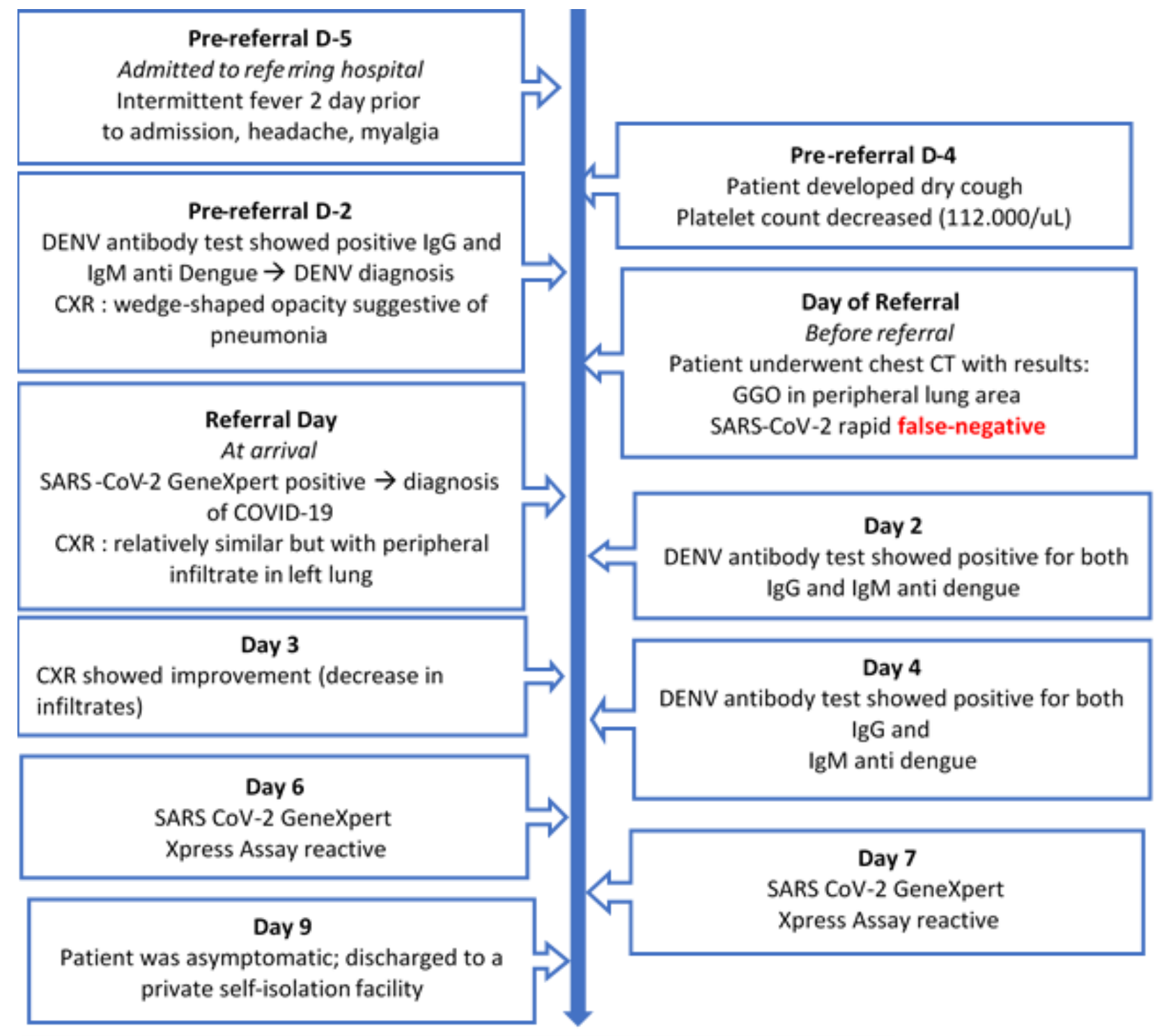

DENV : Dengue Virus, GGO : Ground Glass Opacity, COVID-19 : Coronavirus 19Disease

Fig 3. Timeline of disease

\section{Discussion}

Today, COVID-19 has become a worldwide pandemic. However, Besides the COVID-19 pandemic, Indonesia is also currently facing the dengue outbreaks as the country approaches the rainy season. Hence, it is important for physicians to recognize the possible signs of coinfection of SARS-CoV-2 and DENV in a single patient.

Coinfection of SARS-CoV-2 and DENV may go undiagnosed by physicians, as there is low suspicion toward coinfection even when both diseases are present. This is mainly due to both diseases having a similar disease course, clinical presentations and laboratory profiles. ${ }^{6} \mathrm{~A}$ recent study of 1099 patients with COVID-19 showed $87.9 \%$ patients present with fever, $67.7 \%$ present with cough, and $13.7 \%$ present with headache. ${ }^{7}$ A study by Chen at al. (2020) stated that of 1792 patients with DENV infection, $100 \%$ presented with fever and $25.7 \%$ presented with headache. ${ }^{8}$ However, only $7 \%$ patients of DENV infection presented with cough. ${ }^{9}$ 
A recent meta-analysis showed that the majority of patients with COVID-19 presented with normal hematological test results, increased fibrinogen and CRP levels were the most frequent laboratory findings $\left(55.4 \%\right.$ and $78.4 \%$, respectively). ${ }^{10}$ Leucopaenia and thrombocytopaenia were found in only $15.5 \%$ and $17.6 \%$ of patients with COVID-19, respectively ${ }^{10}$, compared to DENV infection ( $72 \%$ and $49 \%$, respectively), where both findings were very common. ${ }^{9}$ However, both diseases may indeed present with similar laboratory profiles. These results showed that patients with these infections may present with similar symptoms and laboratory profiles, leading to misdiagnosis of both diseases. Such cases have been reported in Singapore, where two patients who previously tested positive for DENV infection were shown to be positive for COVID-19 on further testing, suggesting the first positive dengue test results to be false positives. These Singaporean patients presented with fever, cough, and thrombocytopenia. ${ }^{11}$

In our case, the patient came to the first hospital with fever, myalgia, and thrombocytopenia. The patient's profile was very suggestive of dengue fever. With such a profile, (a positive dengue antibody test, and negative SARS-CoV-2 rapid antibody test,) there was initially little suspicion of COVID-19 at the time of admission. It was only after the patient developed respiratory symptoms the next day and CT-scan showed findings suggestive of COVID-19 that the patient was suspected of the possibility of COVID-19 and referred to our hospital for further evaluation. The patient also presented with mild thrombocytopenia (which had resolved at the time of the referral), slightly elevated fibrinogen and markedly elevated CRP levels, which suggests that the patient indeed had the possibility of SARS-CoV-2 infection from the beginning of admission based on this laboratory profile.

The patient in our report also had a markedly elevated serum ferritin level. Increased serum ferritin level has been observed in COVID-19 and has been linked to the severe form of the disease. ${ }^{12}$ The mechanism of this is still unclear. Some studies have noted cytokine storm as the most likely cause, as ferritin is a well-known marker of inflammation. A wide range of possible causes for increased ferritin (i.e. either by passive release in cell damage or active secretion by macrophages and HepG2 cells) ${ }^{12}$ remains an interesting debate that shall be addressed in future studies.

It is also worthwhile to discuss why the initial SARS-CoV-2 antibody assay showed negative results while subsequent molecular tests were repeatedly positive. We know timing is very important when it comes to rapid (antibody) testing of SARS-CoV-2. Zhao et al. stated that the presence of SARS-CoV-2 antibodies rapidly increased in 15 days after onset $(94.3 \%$ and $79.8 \%$ for IgM and IgG, respectively) compared to less than $40 \%$ in the first 7 days of the disease. ${ }^{13}$ In our case, rapid SARS-CoV-2 test were done on day 7 of the illness, which may explain the negativity of the result due to low amount of produced antibodies.

A recently published study by Lustig et al. found potential antigenic cross-reactivity between SARS-CoV-2 and DENV, which raises the possibility of false-positive DENV antibody testing in those with COVID-19 and vice-versa. This study noted that the structure similarity between the two viruses as the potential cause for the cross-reactivity. ${ }^{14}$ In our setting, the failure to recognize the SARS-CoV-2 infection due to this reason may cause further spread of COVID19 due to false sense of security and lack of isolation.

In conclusion, although there is currently no published data showing increased morbidity or mortality associated with coinfection, delays in diagnosis of coinfection may bring several consequences to the patient and the community. We suggest that at this time of COVID-19 
pandemic, patients presenting with symptoms suggestive of DENV infection in DENVendemic countries should be assessed carefully, particularly when cough is prominent, and leucopenia and thrombocytopenia are not remarkable. We suggest that when the diagnosis of COVID-19 is still uncertain, even when DENV antibody testing is positive, COVID-19 should still be considered and COVID-19 molecular test done without delay. Furthermore, both DENV and SARS-CoV-2 antibody assay results should not be used as the clinical decision-making tools.

\section{Conflict of interest: None}

Ethics: Informed and written consent was obtained from the patient and local ethical committee.

\section{References}

1. World Health Organization, 2020. Novel Coronavirus (2019-nCoV). Situation Report-22, Data as reported by 11 February 2020. Downloaded from: https://www.who.int/docs/defaultsource/coronaviruse/situation-reports/20200211-sitrep-22-ncov.pdf?sfvrsn=fb6d49b1_2

2. Cucinotta D, Vanelli M. WHO Declares COVID-19 a Pandemic. Acta Biomed. 2020;91(1):157160. Published 2020; doi:10.23750/abm.v91i1.9397

3. COVID-19 update in Indonesia. Official site of Indonesia Ministry of Health. Retrieved from: https://infeksiemerging.kemkes.go.id/

4. Biswas, Subhajit, and Soumi Sukla, 2020. COVID-19 Virus Infection and transmission are observably less in highly dengue-endemic cuntries: can dengue vaccines be "repurposed" to prevent COVID-19?. OSF Preprints. April 3. doi:10.31219/osf.io/dzygw.

5. Harapan H, Michie A, Mudatsir M, Sasmono RT, Imrie A. Epidemiology of dengue hemorrhagic fever in Indonesia: analysis of five decades data from the National Disease Surveillance. BMC Res Notes. 2019; 12(1):350. doi:10.1186/s13104-019-4379-9

6. Wu D, Lu J, Liu Q, Ma X, He W. To alert coinfection of COVID-19 and dengue virus in developing countries in the dengue-endemic area Infect Control Hosp Epidemiol. 2020; 1 (Online ahead of print) doi:10.1017/ice.2020.187

7. Guan WJ, Ni ZY, Hu Y, et al. Clinical characteristics of coronavirus disease 2019 in China. N Engl J Med. 2020; 382(18):1708-1720. doi:10.1056/NEJMoa2002032

8. Chen D, Zhang Y, Wu X, et al. A survey of clinical and laboratory characteristics of dengue fever epidemic from 2014 to 2018 in Guangzhou, China. Ann Palliat Med. 2020; 9(1):70-81. doi:10.21037/apm.2019.12.11

9. Damodar T, Dias M, Mani R, et al. Clinical and laboratory profile of dengue viral infections in and around Mangalore, India. Indian J Med Microbiol. 2017; 35(2):256-261. doi:10.4103/ijmm.IJMM_15_423

10. Cao M, Zhang D, Wang Y, et al. Clinical Features of Patients Infected with the 2019 Novel Coronavirus (COVID-19) in Shanghai, China. Preprint. medRxiv.2020; 2020.03.04.20030395. Published 2020 Mar 6. doi:10.1101/2020.03.04.20030395

11. Yan G, Lee CK, Lam LTM, et al. Covert COVID-19 and false-positive dengue serology in Singapore. Lancet Infect Dis. 2020; 20(5):536. doi:10.1016/S1473-3099(20)30158

12. Kappert K, Jahić A, Tauber R. Assessment of serum ferritin as a biomarker in COVID-19: bystander or participant? Insights by comparison with other infectious and non-infectious diseases. Biomarkers. 2020 Jul 18 (just-accepted):1-36. doi: 10.1080/1354750X.2020.179788

13. Zhao J, Yuan Q, Wang H, et al. Antibody responses to SARS-CoV-2 in patients of novel coronavirus disease 2019. Clinical Infectious Diseases. 2020 Jan 1. doi: 10.1101/2020.03.02.20030189

14. Lustig Y, Keler S, Kolodny R, et al.. Potential antigenic cross-reactivity between SARS-CoV-2 and Dengue viruses. Clinical Infectious Diseases. 2020; ciaa1207. doi: 10.1093/cid/ciaa1207 